\title{
Appropriation et productivité linguistiques : comment le locuteur construit-il sa langue?
}

\author{
Bougchiche Redouane \\ Université Paris-Sorbonne (Paris-IV) \\ Laboratoire Sens, Texte, Informatique et Histoire (STIH) \\ redouane.bougchiche@yahoo.fr
}

\begin{abstract}
Résumé. Ce travail propose d'aborder l'un des mécanismes cognitifs agissant sur l'appropriation et la productivité dans les langues : l'analogie. Il vise une interprétation de la productivité et de la manière dont les unités linguistiques sont réalisées en dehors de la théorie générative d'acquisition/apprentissage des langues, en se focalisant essentiellement sur l'acte de langage. On verra que le locuteur ignorant une forme linguistique procède au calcul d'une quatrième proportionnelle afin de répondre au manque de savoirs linguistiques et de produire dans la langue en se basant sur des similarités entre les éléments de l'équation. C'est ainsi que l'apprenant du français langue seconde construit, par exemple, la forme d'un nouveau verbe sans jamais l'avoir rencontrée auparavant. Il pourra inférer, à partir d'un verbe connu descendre : descendu, la forme du participe passé d'un autre verbe, entendre : ?, ? = entendu. Ce serait de cette façon que le locuteur produit dans la langue et avance dans son apprentissage naturel non-guidé. Il use du processus analogique, consciemment ou inconsciemment, tout en s'appuyant sur des exemplaires puisés dans son bassin linguistique. Ainsi, l'analogie est au cœur de l'apprentissage par transmission en situation et non par des règles conscientes. De ce fait, l'appropriation et la productivité linguistiques se trouvent renforcées par ce mécanisme cognitif.
\end{abstract}

\begin{abstract}
This work proposes to analyse one of the cognitive mechanisms acting on learning and productivity in languages: analogy. It interprets the productivity and the way in which the language units are produced, mainly focusing on the speech act, out of the generative theory of language learning and acquisition. The speaker ignoring a linguistic form calculates a fourth proportional to meet the lack of linguistic knowledge, counting on similarities between the equation terms. The learner of Fench as a second language builds, for example, the form of a new verb which he has never met before. He may infer the participle form from a known verb such as descendre : descendu, for an unknown verb entendre :?, ? = entendu. This would be the way followed by the speaker to produce in language and advance in a non-guided natural learning. An analogical process is used, consciously or unconsciously, relying on examplar forms taken from his own language area. Thus, the analogy is at the heart of language learning based on examplars-usage not on rules-usage. Thus, language learning and productivity are strengthened by this cognitive mechanism.
\end{abstract}




\section{Introduction}

La productivité suscite un intérêt grandissant en sciences du langage en raison de la diversité des réalisations, des locuteurs et des mécanismes mis en œuvre dans la langue. Cependant, l'intérêt principal réside dans la cognition du locuteur et les processus mentaux sollicités pour produire et apprendre une langue.

Ce travail s'intéresse au comportement langagier du locuteur apprenant le français comme nouvelle langue et aux processus cognitifs mis en place pour la production des savoirs linguistiques. Parmi ces processus, l'analogie joue un rôle important dans l'appropriation-production linguistique. Travailler sur les constructions analogiques permet d'observer l'organisation cognitive interne grâce à laquelle le locuteur produit et existe dans la langue (Bajrić, 2009). Pour cela, il faudra s'appuyer sur les situations d'emploi discursives avérées et non sur le système, autrement dit, se focaliser sur l'acte de langage (discours) plutôt que sur la langue en tant que système de règles. Car, c'est le locuteur qui construit la langue qu'il utilise, d'où la primauté de l'acte de parole sur l'organisation grammaticale de la langue.

Dans notre contribution, nous nous sommes appuyé sur une étude faite sur des immigrés kabylophones en région parisienne apprenant le français (Bougchiche, 2008), une autre étude concernant l'analogie dans l'apprentissage des langues (Bougchiche, 2013) englobant le corpus des kabylophones et d'autres études sur l'acquisition-apprentissage du français (Bassano (1998b) ; Clark (1998) ; Grégoire (1947) ; HilaireDebove \& Kern (2003), De Heredia (1973); El Kilani (1983) ; Morsly (1983)). Notre corpus ${ }^{1}$ s'appuie sur l'enregistrement de six informateurs, à hauteur de trente minutes chacun, trois «primo-migrants $»^{2}$ n'ayant jamais étudié le français, et trois « jeunes-migrants » ayant suivi une scolarité réduite (jusqu'au collège), et ayant été confrontés à la langue française sur les bancs de l'école algérienne.

A travers ce corpus et ces études, nous avons voulu montrer, en situation d'ignorance, le comportement de locuteurs apprenant le français qui créent et produisent de nouveaux éléments du discours en s'appuyant sur leurs acquis linguistiques.

Dans ce qui suit, nous présenterons d'abord le concept d'analogie en linguistique sans en faire une étude historique. Ensuite, nous aborderons l'appropriation et la productivité linguistiques, ainsi que l'analogie et la productivité chez divers locuteurs apprenant le français comme langue étrangère. Enfin, nous terminerons avec la notion d'erreur linguistique en rapport avec l'analogie. 


\section{L'analogie linguistique}

L'analogie ${ }^{3}$, dans sa forme originale, désigne l'égalité des rapports de grandeurs mesurables (Aristote, 1980). Elle a d'abord été nommée proportion par Pythagore. Suivant la tradition aristotélicienne, l'analogie est constituée de quatre termes $A, B, C$ et $D$, schématiquement, $A: B=C: D$. Autrement dit, la relation qui existe entre les termes $A$ et $B$ est similaire à celle entre $C$ et $D$. C'est cette catégorie d'analogie qui est intéressante à étudier dans le cadre de la production linguistique, car elle permet le calcul d'une inconnue jamais produite par le locuteur à travers ce qu'il connaît de la langue. De plus, elle permet au locuteur d'avoir un appui solide quant au choix des éléments de son discours, et renforce la recherche de similitudes lors du calcul analogique.

Depuis l'antiquité, l'analogie a été au centre des discussions grammaticales, qu'elle s'attache à la conjugaison des verbes, à la nature des mots, ou aux déclinaisons. Les académiciens n'ont pas manqué de signaler son rôle dans la formation du lexique (Dictionnaire de l'académie française, 1798, p. 135). De même, Vaugelas ${ }^{4}$ insiste sur le rôle de l'usage et de l'analogie dans la pratique de la langue qu'il considère :

« comme la copie ou l'image l'est de l'original, ou du patron sur lequel elle est formée, tellement qu'on peut trancher le mot, et dire que notre langue n'est fondée que sur le seul usage ou déjà reconnu, ou que l'on peut reconnaître par les choses qui sont connues, ce qu'on appelle analogie ».

Vaugelas $^{5}$ ajoute que :

«d'un côté, nous avons tout ce qui est attesté ; de l'autre tout ce qui pourrait l'être si l'on suivait les règles de l'analogie. Ainsi, l'usage et l'analogie se complètent-ils parfaitement pour former une image achevée de la langue ».

Les éléments constitutifs de l'analogie proportionnelle peuvent être permutés et le rapport existant sauvegardé. Ainsi, $A$ est à $B$ ce que $C$ est à $D$, s'exprime par $B$ est à $A$ ce que $D$ est à $C$, ou $A$ est à $C$ ce que $B$ est à $D$. Le rapport analogique sera respecté en permutant ces termes comme suit :

1. prendre est à prends ce que mordre est à mords

2. prends est à prendre ce que mords est à mordre,

3. prends est à mords ce que prendre est à mordre.

Cependant, il existe des cas où l'équation se trouve perturbée, comme dans l'exemple (6) :

4. ma mère $:$ moi $=$ ta mère $:$ toi

5. ma mère : ta mère $=$ moi : toi

Cette analogie n'est pas la même dans :

6. *ta mère : $m o i=* m a$ mère $:$ toi $^{6}$ 
Ainsi, l'inversion des éléments d'une proportion est possible pour les analogies formelles, alors qu'elle n'est pas toujours garantie pour les analogies sémantiques ou fonctionnelles.

L'analogie joue un rôle dans la régularisation des formes irrégulières. En ancien français, le verbe trouver prenait à la première personne du singulier la forme je treuve, alors qu'à la première personne du pluriel, il se présentait comme nous trouvons. La forme du singulier a fini par se conformer à celle du pluriel pour devenir je trouve. La même procédure s'est réalisée pour le verbe prouver comme le montre Saussure (1946, p. 222) : «en français, on a dit longtemps : il preuve, nous prouvons, ils preuvent. Aujourd'hui on dit il prouve, ils prouvent, formes qui ne peuvent s'expliquer phonétiquement ».

Aussi, Guillaume (1982, p. 169) considère que «toutes les particules nommées phonétiques sont des analogies ». De plus, il insiste sur le fait «qu'il n'y a pas d'opération constructive du langage qui n'en appelle au tenseur analogique. Le tenseur phonétique isolé, détaché du tenseur analogique, n'existe pas $»^{7}$.

En morphologie verbale par exemple, on distingue les rapports suivants :

- L'analogie dont la similarité est duelle telle que : marcher : je marche = chercher : je cherche, de même que marcher : chercher = je marche : je cherche ;

- La similarité est simple comme dans il marche : il marcha = il voit : il vit ;

- et dont la similarité entre les éléments est nulle telle que il va : il alla = il voit : il vit.

Ajoutons que Paul (1920, p. 107) a insisté sur le fait que le rapport analogique, dans ce type de proportion, doit associer le sens et la forme.

En lexico-sémantique, on peut observer les rapports analogiques dans ce qui suit :

- Un des aspects de cette analogie consiste dans la construction d'éléments qui ne relèvent pas du même paradigme de dérivation affixale. La relation entre champignon et fongique est la même que celle entre relation et relationnel. La première paire de mots n'a pas la même base lexicale, dans la mesure où les mots ne sont pas formellement dérivés l'un de l'autre, contrairement aux deux autres éléments de la proportion : champignon : fongique8 = relation : relationnel. C'est bien le rapport lexicosémantique qui est mis en avant par cette analogie, où fongique sert d'adjectif qualificatif pour champignon, tout comme relationnel l'est pour relation.

- Là où les éléments constitutifs appartiennent au même couple de famille morphologique, autrement dit, avec un rapport formel exprimé entre le moyen et son utilisateur : piano : pianiste = violon : violoniste.

- Le rapport relationnel concerne le sens qui lie les lexèmes tels que : texte : écrire = maison : bâtir. On distingue la relation sémantique entre le verbe et son action sans aucun rapport formel.

- L'analogie lexico-sémantique a une portée plus large qu'en morphologie, par exemple. Il n'y a pas nécessairement besoin de plusieurs points communs entre les éléments de l'analogie pour qu'elle se réalise comme dans eau : (lit de) rivière = circulation : rue. Dans ce cas, c'est la relation instaurée par s'écouler ou contenir qui est mise en avant car, l'eau s'écoule le long d'une rivière ; la circulation s'écoule le long d'une rue, et que la rue contient la circulation, de même que la rivière contient l'eau. 
L'analogie participe pleinement à la production de nouvelles phrases. Elle occupe une place importante dans la simplification de la grammaire. D'après Bloomfield, la construction de phrases relève d'une habitude à pratiquer une opération de substitution. Il suffit, pour un locuteur, d'avoir rencontré une phrase à laquelle il substitue d'autres éléments pour avoir plusieurs autres phrases. Celles-ci sont conçues sur le même modèle sans jamais les avoir entendues auparavant. Pour cet auteur :

\begin{abstract}
« les analogies régulières d'une langue sont des habitudes de substitution. Supposons, par exemple, qu'un locuteur n'ait jamais entendu la forme Donne l'orange à Annie mais qu'il ait entendu ou prononcé une série de formes comme celles qui suivent :

Bébé a faim. Pauvre Bébé! L'orange de Bébé. Donne l'orange au bébé !

Papa a faim. Pauvre Papa! L'orange de Papa. Donne l'orange à Papa!

Bill a faim. Pauvre Bill! L'orange de Bill. Donne l'orange à Bill!

Annie a faim. Pauvre Annie! L'orange d'Annie...............

Il a l'habitude maintenant - l'analogie - d'utiliser Annie dans les mêmes positions que Bébé, Papa, Bill et par conséquent, dans la situation qui convient, énoncera la forme nouvelle Donne l'orange à Annie! »'.
\end{abstract}

Le même procédé s'applique à l'exemple suivant :

- Jean a des parents : Jean rend visite à ses parents $=$

Patrick a des parents : $x ; x=$ Patrick rend visite à ses parents

On voit bien que «le pouvoir explicatif de l'analogie est explicitement étendu à la syntaxe, on ne l'avait envisagé jusqu'ici que pour la morphologie et le lexique » (Lavie, 2003, p. 37).

Les formations par effet de couple (Marchello-Nizia, 2006, p. 85) de syntagmes constituent une autre manifestation de l'analogie syntaxique. En français, avant que est fait sur le modèle de après que, et fonctionne avec le mode subjonctif. C'est également à travers l'analogie que je m'en rappelle a été créé sur le modèle de je m'en souviens.

L'analogie est considérée comme un des moteurs de l'apprentissage-production linguistique, et l'abandon de la notion de règle remet l'analogie au centre des études linguistiques, notamment avec les travaux de Derwin \& Skousen (1989), Itkonen (1997, 2005), Lavie (2003), Monneret (2004), entre autres. Cet intérêt accordé à l'analogie situe le locuteur au centre de l'activité langagière et donne de l'importance aux stratégies cognitives des locuteurs en situation d'apprentissage-production langagière.

Du point de vue cognitif, le processus analogique est sollicité pour deux opérations mentales essentielles : le raisonnement et la résolution de problèmes par analogie (Gentner, 1983 et 1989 ; Goswami, 1992 ; Holyoak \& Thagard, 1995). Les travaux de Gentner (1983, 1989) ont ouvert la voie à de nombreux travaux, sur la compréhension d'une nouvelle situation (cible) sur la base de connaissances précédemment acquises (source). Cette compréhension est celle de la résolution de problèmes à travers la reconnaissance des similitudes relationnelles ou structurelles entre les éléments de l'analogie. Autrement dit, résoudre un problème par la mise en correspondance des éléments de la cible avec ceux de la source.

Sans négliger l'importance des similitudes de surface (celles des attributs), ce modèle est caractérisé par la mise en correspondance (mapping) des relations et des structures entre les situations, domaines et termes de l'analogie. Ainsi, pour construire une analogie, on apparie les relations entre la source et la cible. Gentner parle ainsi de l'appariement structurel (structure-Mapping-Engine). 
Pour Gentner, deux principes interviennent dans la mise en correspondance des structures et relations de la source et de la cible: le principe de systématicité qui représente le transfert d'un ensemble cohérent de relations de la source à la cible ; et le principe de transparence qui représente le degré de ressemblance perceptive entre les termes de la situation source et ceux de la situation cible.

Ainsi, l'analogie est au centre des activités mentales. D'une part, l'homme n'aborde l'inconnu qu'à travers le connu. D'autre part, l'homme cherche toujours des solutions à ses problèmes en s'appuyant sur les situations anciennes qui lui fournissent le matériel de son raisonnement, et les solutions à ses problèmes. C'est donc un processus heuristique dont l'homme ne peut se passer.

\section{Appropriation et productivité linguistiques}

L'analogie est également un des processus ${ }^{10}$ intervenants dans la construction du langage. Tomasello (2003) rattache ce processus à celui de la schématisation qu'il considère comme une forme d'analogie, celle-ci étant plus abstraite (Tomasello, 2003 : 164 ; 298). A travers ce processus analogique, on comprend comment les locuteurs créent et produisent dans la langue en fonction de ce qu'ils ont entendu dans leur communauté linguistique. Le locuteur développe sa capacité à imiter les locuteurs experts, non seulement dans la forme du discours, mais aussi dans l'intention de communication (Tomasello, 2003). Pour communiquer dans la langue, le locuteur produit ses propres discours en construisant des schèmes servant de base analogique à de nouvelles constructions. C'est cette position qui est suivie dans ce présent travail.

Cette capacité mentale met en avant la faculté du locuteur d'être productif dans la langue. Et comme le souligne Lavie (2003 : 15), « la productivité est le problème principal de la linguistique. Rendre compte de la productivité est, pour la linguistique, une tâche centrale ». Pour être productif dans la langue, soit le locuteur se base sur des règles toutes faites, soit il mobilise des connaissances déjà mémorisées présentes à son esprit. C'est dans le cadre du second modèle que l'analogie est intéressante à étudier car, d'une part, le locuteur ignore les normes grammaticales de la langue qu'il apprend, et d'autre part, c'est en s'appuyant sur les situations d'apprentissage spontané non-guidé que le langage humain a été construit, et qu'en l'absence des institutions scolaires, les locuteurs apprennent leur(s) langue(s) par transmission en situation et non par règles conscientes.

Malgré que tout nouvel énoncé soit présumé inédit, le locuteur s'appuie sur des éléments déjà acquis pour produire dans la langue. Le locuteur, ignorant une forme linguistique qu'il veut produire, cherche dans ses connaissances celles dont la ressemblance le guide à produire la nouvelle forme. Il pose les éléments de la langue deux par deux, et cherche à construire le terme inconnu de manière à avoir : $\mathrm{A}: \mathrm{B}=\mathrm{C}: X$, dont $X$ = D. Par exemple, un enfant de cinq ans, connaissant le couple méchant : méchanceté, a produit *gentisté (gentillesse) sur gentil (Grégoire, 1947, p. 73). Cet enfant maîtrisant les antonymes méchant et gentil, en rencontrant méchanceté, a produit son opposé *gentisté selon le même modèle morphologique. Ainsi, il a calculé, consciemment ou inconsciemment, une inconnue à partir de ce qu'il connaît, en posant une analogie à quatre termes, dont trois connus et un ignoré.

Dans cette perspective analogique, le locuteur s'appuie sur des exemplaires (Lavie, 2003 ; Tomasello, 2003) qui sont l'ensemble des savoirs linguistiques rencontrés sous toutes leurs formes, et mémorisés sous forme d'empreintes linguistiques pour produire et comprendre de nouveaux énoncés (Lavie, 2003 ; Bougchiche, 2013). Lavie (2003: 9) soutient l'idée que «la productivité est donc la possibilité de produire ou comprendre une infinité d'énoncés dans un cadre linguistique donné, c'est-à-dire à "compétence" constante ». Selon cet auteur, la productivité linguistique est la combinaison de la productivité structurelle et la productivité systémique. 
Pour Lavie :

«la productivité linguistique dans son ensemble résulte du jeu combiné de la
productivité structurelle et de la productivité systémique. [...] La productivité
structurelle couvre la morphologie et la syntaxe en continuité» ${ }^{11}$,

la productivité systémique est relationnelle, elle se base, non sur les ressemblances formelles entre les unités linguistiques, mais sur la relation qui lie ces unités entre-elles.

Dans le cadre de l'appropriation linguistique, on peut comprendre que le locuteur procède à des productions structurelles, car il accède aux savoirs linguistiques par rapprochement des différents éléments formels qu'il reçoit. Puis, avec le développement de sa compétence linguistique, il accède au stade de productivité systémique où il ne se focalise pas seulement sur les ressemblances morphologiques, syntaxiques, etc. pour produire dans la langue, mais il opère des ponts entre les savoirs acquis pour construire des analogies sans ressemblances formelles, telles que les analogies cognitives et lexico-sémantiques ta mère $:$ toi = ma mère $:$ moi ; il va $:$ il alla = il voit $:$ il vit champignon $:$ fongique = relation : relationnel $;$ texte $:$ écrire $=$ maison $:$ bâtir , etc.

Le locuteur développe ainsi une nouvelle compétence qui lui permet d'accéder à un autre niveau de production dans la langue sans pour autant se focaliser uniquement sur les similarités formelles. Ainsi, il passe de marcher : je marche = manger $:$ je mange à il est $:$ je suis = il va: je vais ou j'irai $:$ je vais = je mangerai : je mange, entre autres.

C'est à travers l'analogie systémique que les dernières constructions sont possibles. Dans le cadre d'une productivité structurelle, le modèle suivi en morphologie, par exemple, est le suivant : base verbale + flexion $=$ forme verbale fléchie. Si cette forme convient à beaucoup de constructions analogiques, elle présente certaines limites quand il s'agit des verbes à base verbale différente (allomorphes), selon le temps exprimé par exemple : irai est à vais comme mangerai est à mange. Cet exemple se répète avec les verbes être et aller tels que : êtes est à suis ce que allez est à vais. Il en est de même pour les constructions lexico-sémantiques telles que : écrire est à texte comme bâtir est à maison.

Dans ces exemples, le locuteur met en relation des éléments de la langue qui n'ont pas la même forme. Il connecte entre eux des mots différents dont la relation est basée sur le sens ou sur une racine verbale différente. Parce que le locuteur ne connaît pas la composition de la langue en sous-catégories, en verbes à trois groupes différents, et en champs lexicaux variés, il fournit un effort cognitif supplémentaire afin d'accéder à ces distinctions, et de pouvoir réaliser des constructions systémiques.

Ainsi, l'appropriation linguistique est incrémentale. Le locuteur apprend la langue par paliers, et les savoirs par paradigmes, puis accède au niveau systémique où il associe les savoirs deux par deux de sorte à relier les unités linguistiques entre-elles sans rapports formels, mais que les différentes compositions verbales, lexico-sémantiques et cognitives permettent. C'est donc à travers les constructions analogiques (structurelles et systémiques) $^{12}$ que le locuteur produit dans la langue pour pallier au manque de savoirs linguistiques et avancer dans son apprentissage. Celui-ci se base sur les exemplaires reçus de la masse des discours d'autrui. C'est également à travers cette combinaison que le locuteur est créatif, car pour résoudre une équation analogique et trouver l'élément manquant à sa production, il crée une forme qu'il ignore. Cela confère à l'analogie la place de processus heuristique, essentiel à l'appropriation-production linguistique. Ce dont le locuteur a besoin dans sa pratique linguistique, c'est d'arriver à exprimer dans la langue ce qui est possible et ce qui ne l'est pas (Lavie, 2003 : 17). Pour faire face à des situations de communication inédites, il s'appuie sur les anciennes connaissances qu'il maîtrise déjà, et c'est en se focalisant sur ces connaissances que l'analogie prend forme et que le locuteur devient productif. 


\section{Analogie et appropriation d'une nouvelle langue}

L'analogie comme processus de production vaut également pour le locuteur apprenant une nouvelle langue, cette fois, dans des conditions différentes. En plus des connaissances nouvellement acquises, la langue maternelle joue un rôle dans les productions de la nouvelle langue.

Nous avons vu que la productivité langagière s'appuie sur les expériences linguistiques antérieures des locuteurs qui permettent une production automatisée de modèles tout faits (exemplaires) adaptés aux différentes situations de communication. Ces exemplaires servent à construire de nouvelles productions jamais réalisées, autrement dit, des connaissances en construction. C'est dans le processus analogique que se trouve la clef du fonctionnement du locuteur que ce soit dans un cadre monolingue, ou dans un cadre bilingue.

En intégrant les connaissances linguistiques rencontrées durant les différents contacts avec autrui, le locuteur apprenant le français imite les formes et les structures linguistiques fournies par ses interlocuteurs (Tomasello, 2003). Cela passe par plusieurs étapes. D'abord, le locuteur rapproche les nouvelles données linguistiques qu'il entend de ses primo-savoirs afin de les comprendre. Ensuite, dans le cadre de nouvelles productions, il s'appuie sur ces exemplaires afin de trouver le modèle adéquat pour une production personnelle. Dans cette démarche, il s'agit, pour le locuteur, d'une création. Enfin, si cette dernière n'est pas rejetée par les interlocuteurs, elle finira par intégrer les paradigmes, ou les réseaux de savoirs préconstruits. C'est ainsi qu'il conjuguera un nouveau verbe qu'il rencontre, par exemple coudre, à la même forme que le verbe moudre dont il détecte une certaine similarité phonétique (ou que les deux verbes ne se différencient que par l'initiale $[\mathrm{k}]$ et $[\mathrm{m}])$ : moudre $:$ je mouds = coudre $:$ je $x, x=$ je couds (Bougchiche, 2008). Il en fera de même lorsqu'il s'agira de former le pluriel des noms masculins. Il appliquera la forme la plus utilisée à l'oral pour le reste des noms pluriels.

En apprenant une nouvelle langue, le locuteur se construit des modèles mentaux pour représenter chaque catégorie du système linguistique. Ainsi, il construit des schèmes verbaux sous forme de $X+/ \varepsilon /$ «-ais »; ou $X+/ \tilde{J} /$ «-ons », etc. (Tomasello, 2003). Ensuite, il se sert de ces modèles pour produire de nouvelles unités. A chaque fois qu'il rencontre une nouvelle forme verbale, il lui construit un modèle représentatif qui servira à de nouvelles constructions analogiques. C'est de cette façon qu'il a produit je travaillais sur le modèle de je marchais (Bougchiche, 2008).

Aussi, il pourra se focaliser sur une similitude formelle (ressemblance de signifiants : les faux-amis, entre le français et l'espagnol par exemple) et construire une analogie lexico-sémantique, malgré que la différence sémantique provoque l'incompréhension du discours chez l'interlocuteur. Ainsi, aclarar est utilisé par un hispanophone pour rendre son équivalent en français éclairer, alors qu'il véhicule un autre sens : éclaircir. L'encodage phonologique peut faciliter l'apprentissage et la mémorisation des lexèmes, et dégager des similitudes dans les énoncés, mais cette action induit en erreur (généralement sémantique). Le locuteur adapte le lexique du français éclairer au modèle de l'espagnol aclarar sur la base d'un autre mot, adivinar, qu'il maîtrise et dont le sens est identique dans les deux langues : « deviner ». Cela l'amène à répéter la même action par analogie avec d'autres mots qui, selon lui, se prêtent aux mêmes adaptations.

Pour apprendre le lexique, le locuteur s'appuie sur des mots-clés afin d'associer les mots nouveaux à ceux déjà appris. À ses débuts, lorsque les langues en contact sont de même famille (le cas du français et de l'espagnol), il mémorise les formes lexicales et construit la dimension sémantique à partir du sens qu'il connaît du lexique de la langue maternelle (désormais L1), puis intégrera les spécificités sémantiques de la nouvelle langue (désormais L2). Cette forme est intermédiaire car elle ne représente pas fidèlement le sens véhiculé par le mot de la L2. La représentation du mot appris sera modifiée soit par ajout des différents traits sémantiques manquants, soit par l'attribution du sens adéquat et modifier les erreurs (le cas de aclarar et éclairer, par exemple). Dans tout apprentissage d'une nouvelle langue, le locuteur a besoin de traiter du sens. Il analysera les données linguistiques inconnues en se basant sur ce qu'il connait 
déjà, à la fois de la L1, et de la L2 qui sont ses connaissances personnelles dont il se sert pour compenser ses lacunes.

Au départ, la L1 représente l'arrière-plan sémantique de la L2 comme l'a exprimé Vigotsky qui considère que :
«la langue maternelle et les concepts spontanés (la dimension sémantique de la langue maternelle) constituent l'arrière-plan et la prémisse de l'apprentissage des concepts non spontanés (concepts scientifiques) et des langues secondes $»^{13}$.

Que la généralisation soit lexicale ou sémantique, l'analogie avec la L1 permet d'abord au locuteur d'avoir les moyens de combler des lacunes lexico-sémantiques, ensuite de créer un nouveau système conceptuel pour exister dans la nouvelle langue. Ce dernier point est souvent source de conflits (d'interférences) entre les systèmes conceptuels des deux langues en question.

Un autre aspect des productions analogiques en français se trouve dans la néologie. Le locuteur procède à des créations de nouveaux mots à partir de ce qu'il a appris. Un kabylophone qui a appris à former les noms d'agents par affixation d'un morphème productif - eur ${ }^{14} \mathrm{ou}$-iste, pour les noms de métiers, produira de nouveaux lexèmes pour cette catégorie. Ayant appris le lexème plongeur formé à partir du nom commun (la) plonge, le «jeune-migrant» forme un schème productif $X+$-eur et $X+$-iste, et construit à partir de taxi le terme *taxieur, de même qu'il produit hittiste ${ }^{15}$ [hițist] pour hitt «mur » à partir de pompiste qu'il envisage dans les proportions suivantes : plonge : plongeur = taxi : taxieur; pompe : pompiste = hitt $:$ hittiste $;$ de même que hitt $:$ hittiste = houma « quartier »: houmiste ${ }^{16}$ (Bougchiche, 2008 ; Queffélec et al., 2002).

La créativité lexicale analogique est un élément naturel qui sert le locuteur et la langue. Ils reflètent d'abord l'influence du français standard sur la construction lexicale du français régional, en l'occurrence celui d'Algérie ; ensuite, ils démontrent l'influence des premiers lexèmes analogiquement construits en français d'Algérie sur d'autres lexèmes de cette même variété. Cette procédure se renouvelle avec d'autres morphèmes, en l'occurrence -age, dans d'autres formations lexicales telles que : dégoûter qui a donné * dégoûtage (Bougchiche, 2008), sur le modèle de $V$ (radical verbal) + -age comme dans démarrer : démarrage. Ces productions dues aux particularités régionales du français d'Algérie (Queffélec et al., 2002), et du Maghreb en général, ne nuisent aucunement à l'apprentissage du français standard, et au respect de ses particularités que nous retrouvons dans les discours de locuteurs apprenant le français en région parisienne.

Le kabylophone apprenant le français utilisera les verbes dans leur forme infinitive, simple à reproduire pour construire des phrases. Cet usage est facilité par ses interlocuteurs qui s'adressent à lui avec des formes verbales simplifiées ${ }^{17}$. Le locuteur natif présente le verbe à l'infinitif à l'apprenant lorsque ce dernier ne peut pas construire un énoncé avec le verbe conjugué. Le "primo-migrant », ayant intégré des structures telles que Radical verbal $+/ e /+$ Radical verbal $+/ e / ;$ Radical verbal $+/ e /+N$; toi porter $+X$, toi donner $+X$; toi donner $+X+m o i$, produit des énoncés tels que : aller manger, toi porter sac, toi donner argent moi, etc.

La prise de conscience de la possibilité que l'action soit exprimée différemment dans le temps, fera que le locuteur contextualise les formes les plus fréquentes pour l'usage qu'il souhaite exprimer. Durant l'interaction avec autrui, l'apprenant demande souvent aux autres locuteurs de lui indiquer comment il peut exprimer un fait linguistique dans la nouvelle langue par «comment on dit.....». Le «jeunemigrant » construit par là des modèles tels que : il est où $X$ ?; où est $X ?, j$ 'ai + pas + radical verbal $+/ e /$, et autres, sur la base d'exemplaires rencontrés tels que : il est où Djamel ? Où est la clef? J'ai pas mangé, etc. Le même procédé s'observe dans d'autres langues, ce qu'on trouve chez Tomasello (2003) pour l'anglais sous forme de $I$ wanna $X$, let's $X$, etc. Le savoir fourni renforce sa construction et son rappel lors d'un futur usage. Le locuteur crée des catégories pour chaque forme linguistique rencontrée pour l'utiliser dans de futures productions jamais entendues. 
Pour une action passée, le kabylophone a utilisé la forme la plus fréquente à l'oral : le passé composé. À chaque fois qu'il rencontrera un nouveau verbe, il reprendra cet usage fréquent. Cette forme serait d'abord mémorisée et intégrée au nouveau système verbal, certes incomplet, mais déjà en place ; ensuite, elle serait reprise pour produire de nouvelles constructions pour de nouveaux verbes. La même procédure serait remarquée dans l'apprentissage de l'imparfait que les locuteurs natifs utilisent. Les premiers énoncés servent à construire un paradigme des formes verbales représentant les actions passées (au passé composé et à l'imparfait). C'est ce que nous avons relevé dans les énoncés des «jeunes-migrants », comme suit :

- J'ai cherché travail

- J'ai parlé mon patron

- J'ai travaillé dans le bâtiment

- J'ai pas le temps

- Je suis fatigué

- Je suis parti à Lyon

- Je suis pas à la maison

Ces énoncés feront que le locuteur intègre cette forme verbale simple $j$ 'ai + Radical $+/ e /$ et la forme je suis + radical +le/ (participe passé des verbes en -er), ou je suis + adj et je suis + pas + CCL.

De même pour les formes à l'imparfait comme dans :

- J'avais une maison à Béjaia

- J'avais pas l'argent

À travers ces exemples, le locuteur apprend à la fois les formes des temps verbaux et l'usage des auxiliaires être et avoir.

Pour comprendre le procédé suivi par le locuteur dans l'apprentissage syntaxique en L2, on peut prendre l'exemple de la phrase simple du français $(\mathrm{S}+\mathrm{V}+\mathrm{O})$ apprise par un kabylophone : je prends le métro. Cet énoncé est récurrent dans les discours quotidiens des travailleurs immigrés de la région parisienne. En fonction des occurrences, ce locuteur abandonne la structure qu'il utilisait au début de son apprentissage du français, à savoir : moi attraper mitro ${ }^{18}$, due à la version kabyle comme suit (Bougchiche, 2008):

«tsatafa $\gamma$ amitro »

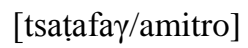

littéralement «j'attrape (habituel, duratif) métro »

sémantiquement «je prends le métro »

Le locuteur substitue d'abord le verbe de la phrase prendre à attraper, puis intégrera l'article défini qui détermine le mot métro pour enfin réaliser la forme correcte je prends le métro. Cette production est déterminée par les divers contextes, où le locuteur relève les différents emplois du mot métro dans le discours d'autrui tels que : tu prends le métro ligne 4 jusqu'à Châtelet, puis la ligne 1 jusqu'à Bastille. Dans un autre contexte, il entendra $j$ 'ai pris le métro mais y avait un accident sur la ligne, etc. Il se rendra compte qu'on n'attrape pas le métro mais qu'on le prend. L'apprentissage combinatoire de la structure française a d'abord permis au locuteur d'améliorer la production de cet énoncé ; ensuite de passer d'une analogie avec la L1 à une autre avec la L2 (attrape ma main devient prends ma main (Bougchiche, 2008) $)^{19}$, plus bénéfique pour l'intégration et l'organisation des constituants grammaticaux du français, 
comme l'article défini, absent du système linguistique du kabyle. C'est en suivant cette démarche que le locuteur construit son discours dans la nouvelle langue, à partir des premiers usages des structures syntaxiques adéquates.

On déduit que le locuteur se base sur les interprétations de précédents énoncés pour en former et en comprendre de nouveaux. Si le locuteur détecte des similitudes et des correspondances entre le(s) nouveau(x) et les anciens énoncés, la démarche (processus analogique) est alors renforcée, la représentation syntaxique aussi. Dans le cas contraire, le locuteur abandonne le premier énoncé (attraper main), et en cherche un autre plus compatible avec la nouvelle construction, ce qui appuie l'idée que le locuteur abandonne une analogie pour se tourner vers une autre qui servira mieux son besoin expressif. L'analogie est au cœur des constructions syntaxiques dont la source se trouve dans les différents exemples énonciatifs antérieurement acquis.

Ainsi, le locuteur construit une représentation syntaxique productive. Il rapportera chaque nouvel énoncé à la structure syntaxique de productions dont les résultats étaient efficaces, il en déduira que sa production répond aux critères syntaxico-sémantiques de la nouvelle langue. Poursuivant son apprentissage, il se base à la fois sur les énoncés des locuteurs natifs et ceux de ses pairs (immigrés, par exemple) pour intégrer de nouvelles combinaisons de la nouvelle langue. Les acquis servent de base à de nouvelles productions et apportent un fondement structurel et sémantique, certes incertain, mais dont l'efficacité a été observée dans de précédentes constructions. De ce fait, l'analogie peut représenter, à chaque fois, le circuit productif qu'emprunte le locuteur tout au long de son appropriation linguistique.

Ces remarques sont observables particulièrement durant l'apprentissage « sur le tas ». Tout nouveau savoir sera intégré, modifié et assimilé aux données déjà apprises et aura un usage systématique (Bouton, 1969) par analogie avec d'autres savoirs similaires. Le locuteur trouvera dans ses acquis le matériel pour créer de nouveaux énoncés, faire évoluer sa langue en intégrant le maximum de structures possibles.

Par ailleurs, il existe des situations où le savoir linguistique est peu productif, le cas des expressions figées et idiomatiques par exemple. Dans le cas où un locuteur rencontre l'énoncé suivant : ci-gît dont il comprend la signification, il reprendra cet exemple pour produire un seul et unique type d'énoncé par analogie. À l'instar de certaines expressions françaises comme : ci-gît, chaque langue possède des tournures dont l'utilisation est limitée, que le locuteur emploie uniquement dans des contextes spécifiques tels que : ci-gît monsieur $X$ où seul le nom du défunt diffère, ci-gît monsieur $X, Y, Z$, etc. par une reprise intégrale de l'expression sur une épitaphe. Ces expressions figées ne permettent pas au locuteur de construire de nouveaux contenus sémantiques, elles sont peu/non productives. On conclut que, dans certains cas, l'analogie permet une reproduction restreinte des structures spécifiques.

L'appropriation d'une nouvelle langue permet au locuteur de développer des capacités cognitives supplémentaires qu'une seule langue n'aurait pas permis d'avoir, ce qui lui permet de développer une « pensée analogique ». L'analogie permet au locuteur de s'initier à la nouvelle langue en découvrant son fonctionnement à travers des exemplaires puisés directement dans son bassin linguistique le plus proche.

Se mouvoir au sein d'une autre langue en luttant contre l'arrivée et l'influence de la langue maternelle, c'est une manière de résister aux analogies interlinguistiques, et passer aux analogies intralinguistiques. Le locuteur observant le fonctionnement de la langue maternelle peut envisager et anticiper celui de la nouvelle langue. Il saura, à chaque fois, qu'il existe des possibilités de construction des éléments de cette nouvelle langue, à l'instar de ce qui existe dans la langue qu'il maîtrise. En d'autres termes, il émettra l'hypothèse que, dans cette langue, il existerait des tableaux de conjugaison où il pourrait conjuguer des verbes par analogie avec ceux déjà appris. Il saura également qu'il existerait des possibilités de constructions lexicales communes à un nombre illimité de mots, et qu'il pourrait créer de nouvelles formes de la même façon qu'il le faisait avec d'autres formes de la L1. Enfin, il sait que les constructions syntaxiques répondraient à un ordre qu'il peut reproduire par analogie. Le recours à la langue maternelle, en dépit du résultat attendu et des doutes suscités, reste une étape essentielle lorsqu'on apprend une nouvelle langue. 
L'influence d'une langue sur une autre ne se limite pas à la L1, mais à toutes les langues que le locuteur maîtrise ou tente de maîtriser, d'où la nécessité de parler de compétence analogique interlinguistique. Cette dernière permet au locuteur de passer d'une langue à une autre, et de se servir des éléments de l'une pour construire un savoir dans l'autre, de troisième ou de quatrième rang, sans pour autant négliger la première et la seconde. En revanche, son choix s'effectue en fonction des correspondances qu'il juge efficaces, ou bien, dont il a observé l'efficacité du résultat. Cette activité est très complexe et intellectuellement coûteuse pour le locuteur, mais indispensable à son développement linguistique et communicationnel. L'analogie plurilingue ne se limite pas aux influences des deux premières langues apprises, elle concerne toutes les langues en présence.

Nous avons observé l'intérêt porté au passage d'un modèle structurel de la langue maternelle à celui de la nouvelle langue, et le recours aux analogies dans les productions syntaxiques interlinguistiques et intralinguistiques. Le contraste entre les deux systèmes s'estompe progressivement, et la période d'intégration et d'accommodation des savoirs appris est riche en analogies intralinguistiques. La progression dans l'apprentissage fera que le locuteur abandonne le recours aux analogies avec la langue maternelle au profit du système linguistique de la nouvelle langue. En d'autres termes, dès que le locuteur accède à suffisamment d'éléments de la nouvelle langue, leurs influences dépasseraient celle de la langue maternelle. Dès lors, l'analogie suit un développement naturel qui passe de l'inter- à l'intralinguistique, ce qui atteste également de la maîtrise conceptuelle de la nouvelle langue à un stade avancé de l'apprentissage. Cependant, les constructions analogiques sont également sources d'écarts linguistiques.

\section{Productivité et erreurs linguistiques}

Tout apprentissage et toute production langagière par analogie ne mènent pas nécessairement à une production admise par les autres locuteurs. Qu'est-ce qui explique la «défaillance » de ce type de production? L'erreur est-elle due au processus engagé dans sa production ? Cette «maladresse » productive est-elle une tare pour la construction linguistique?

Chez l'enfant, comme chez l'adulte, le fait de reproduire les formes apprises et de les généraliser à d'autres provoquerait des écarts, car la langue n'est pas régulière dans son organisation et dans ses formes. Ces écarts peuvent être répartis en deux catégories, ceux qui relèvent d'une mauvaise maîtrise du système linguistique (erreur), et ceux qui relèvent d'une mauvaise réalisation du savoir maîtrisé (faute). L'erreur analogique suit le même procédé cognitif sollicité pour la production des formes linguistiques attestées. La seule différence est que celles-ci correspondent à ce qui est recommandé, et que celle-là donne un résultat qui n'est admis par les interlocuteurs. L'écart est involontaire car, le locuteur ne peut pas savoir que son discours est erroné étant donné qu'il le produit conformément à ce qu'il connaît de la langue, que ce qui peut être considéré comme écart par la norme, peut ne pas l'être dans l'usage (discours), et que les réalisations analogiques sont des créations nouvelles pour le locuteur.

Les erreurs représentent des créations que les interlocuteurs peuvent tolérer si elles n'affectent pas la communication. C'est parce qu'on crée dans la langue qu'on fait des erreurs. De plus, à l'oral, on se focalise plus sur le contenu sémantique du message qu'à sa forme. Ainsi, il faudra situer la création analogique au niveau de l'acte de langage, et prise en synchronie ${ }^{20}$. Des exemples comme *ils croivent au lieu de ils croient sur le modèle de ils boivent (boire : ils boivent = croire : *ils croivent) ; *aller au coiffeur au lieu de aller chez le coiffeur sur le modèle de aller au marché, etc., (également faits de locuteurs francophones natifs), sont nombreux et tolérés, car ils n'affectent pas l'intercompréhension et ne gêneraient pas les interlocuteurs qui les entendent. 
Aussi, la conjugaison des verbes irréguliers est rapprochée de celle des verbes réguliers. A l'oral, l'apprenant unifie, par exemple, les formes des verbes aller et avoir à la première personne du singulier dans *je va /*j'ira; *c'est moi qui l'a fait, *c'est moi qui va (Bougchiche, 2008) ${ }^{21}$ comme il a l'habitude de le faire pour les verbes réguliers du premier groupe en /e/. Ces exemples ont été produits par analogie avec la forme de la deuxième et de la troisième personnes du singulier comme suit : tu vas, il va, *je va; tu iras, il ira, *j'ira; c'est toi qui l'as fait, c'est lui qui l'a fait, *c'est moi qui l'a fait. Le locuteur applique la forme la plus fréquente (qu'il entend) pour le verbe avoir à la deuxième et à la troisième personnes du singulier, tu vas et $i l v a$ à la première personne du singulier.

Dans ces productions, il s'appuie sur une forme verbale maîtrisée, celle du verbe monter par exemple, pour produire le verbe aller ${ }^{22}$ comme dans : tu montes : je monte = tu vas: *je va. La forme, *je va au travail, peut s'observer chez la plupart des locuteurs apprenant le français (kabylophones, germanophones, hispanophone, russophones, etc.). L'explication de ces erreurs se trouve également dans la difficulté cognitive à mémoriser les différentes variantes verbales d'un même verbe. Ces formes présentent « un défi à la mémoire », à l'instar de : je vais, tu vas, vous allez, j'irai, que j'aille, en face de : je chante, tu chantes, vous chantez, je chanterai, que je chante, et que «seules la haute fréquence d'emploi et la contrainte collective réussissent à maintenir de telles anomalies » (Frei, 1993, p. 28). En d'autres termes, si l'utilisation de ces formes verbales n'était pas aussi fréquente, elles auraient été régularisées comme les autres formes irrégulières (l'exemple de je treuve devenu je trouve).

La généralisation du pluriel régulier non marqué, sur les pluriels irréguliers en -al est une source d'erreurs. La ressemblance de la finale en -al des mots au singulier favorise l'écart entre les formes régulière et irrégulière. Le locuteur produit des pluriels impropres : *des chevals, *des animals et *des journals sur la base des autres pluriels non marqués une voiture : des voitures. Ce pluriel reste le seul phonétiquement reconnaissable, car il ne ressemble pas à la forme du singulier (le reste des pluriels en - $S$ ou $-x$ n'est repérable qu'à l'écrit, la forme du singulier étant la même que celle du pluriel et que le morphème du pluriel n'est pas prononçable). C'est d'ailleurs l'une des raisons qui poussent le locuteur à produire des analogies fautives telles que *chevals. À l'oral, le pluriel est marqué par les déterminants définis ou indéfinis : les et des; les démonstratifs pluriel : ces; les pronoms possessifs : mes, nos, etc. Ce pluriel particulier nécessite un apprentissage complémentaire pour le distinguer des autres formes. Le nouvel apprentissage servira de base analogique pour la formation du pluriel de mots en -al nouvellement rencontrés. À l'oral, le singulier ne se distingue pas du pluriel, ce qui favorise ces erreurs, du moins pour le cas de cheval et journal.

L'influence de la L1 est très présente durant les premières étapes de l'apprentissage du français. Un lusophone apprenant le français a produit sécheur ${ }^{23}$ (de cheveux) pour sèche-cheveux à partir de sécher par analogie avec ce qu'il connaît du portugais, à savoir secar « sécher » : secador " sécheur » qu'on peut analyser comme suit : secar $:$ secador = sécher $:$ sécheur. Un germanophone apprenant le français ayant pour habitude d'attribuer le genre féminin à tout ce qui concerne la femme et le genre masculin à tout ce qui relève de l'homme, des saisons, et du temps, transpose les caractéristiques du genre de l'allemand à celui du français. Il produit *le pluie par analogie avec Der Regen ${ }^{24}$ (la pluie). Le recours aux formes de la L1 se réalise en cas d'ignorance du genre du mot en français.

Le fait qu'un locuteur produise une erreur sur la base d'une forme connue est une solution envisageable, attendue, et somme toute, moins négative qu'une invention d'une forme correcte dont il ne maîtrise pas le mécanisme de formation. Autrement dit, il est très difficile de voir un locuteur produire la forme du passé simple du verbe être : fut, s'il ne l'a jamais entendue, alors qu'il peut produire *goed, en anglais, car il connaît l'usage du morphème producteur du passé et du participe passé - $e d$, très fréquent, comme dans like: liked. Dans le premier cas, le locuteur ne dispose pas de support de conjugaison du verbe être, à racines multiples, dont l'apprentissage nécessite un effort intellectuel considérable, et surtout une première rencontre de toutes les formes correspondantes; tandis que dans le second cas, il lui suffit de poser une analogie s'appuyant sur les formes disponibles en mémoire. Cette difficulté est moindre pour le locuteur qui produit une forme à partir de ce qu'il a déjà vu et connu, et que la tâche est presque impossible, pour un locuteur ordinaire, quand il s'agit d'inventer une forme correcte ex nihilo. 
Dans un cadre bilingue, on ne peut pas résumer les erreurs seulement aux analogies avec la langue maternelle ; elles s'appliquent aussi aux constructions inappropriées, formées à partir des savoirs de la nouvelle langue, et ce en contradiction avec la conception de l'analyse contrastive qui considère que les écarts linguistiques, durant l'apprentissage d'une nouvelle langue, sont dus essentiellement à l'influence de la langue maternelle (Bange, 2005). Ainsi, le locuteur traite à la fois les éléments de la langue à apprendre et ceux de la langue apprise dont il doit gérer l'influence.

L'erreur est perçue comme un écart par rapport à ce que la langue autorise. Or, dans le cadre d'un apprentissage naturel, l'erreur analogique est une construction faite à l'image d'une autre dont le résultat est hasardeux et que le procédé de formation a été respecté. La généralisation analogique est bénéfique quand le système linguistique est régulier, tandis que l'irrégularité favorise l'erreur. C'est parce que le système est organisé différemment qu'une création analogique peut être maladroite et provoquer des erreurs (inévitables).

De plus, ce qui explique l'écart linguistique est le fait que le locuteur s'appuie sur son apprentissage structurel, donc des tableaux et des formes fréquentes rencontrées tout au long de son apprentissage. L'apprentissage systémique, qui est une étape plus avancée dans cette activité cognitive où le locuteur ne se focalise pas seulement sur les ressemblances formelles pour produire dans la langue, mais procède à des rapprochements linguistiques pluridimensionnels. Cela lui permet d'éviter des erreurs dues à l'application d'une forme apprise (déclinaisons, flexions, etc.) sur une autre qui ne correspond pas à la nouvelle construction. C'est notamment le cas des marquages morphologiques, des déclinaisons verbales entre les différents groupes verbaux du français.

\section{Conclusion}

Après un positionnement théorique qui mettait en avant un mode de production langagière et l'importance des relations analogiques dans l'apprentissage du français langue étrangère, cet article a proposé un cadre qui permet de découvrir les propriétés de l'analogie agissant sur la cognition humaine, dans sa forme linguistique. L'observation faite démontre que l'analogie est à l'œuvre dans le fonctionnement langagier du locuteur et de l'organisation de la langue. L'explication des résultats d'une approche constructrice d'une langue passe, nécessairement, par le raisonnement et la résolution de problèmes par analogie. Le locuteur trouve, dans la somme des expériences linguistiques vécues et des savoirs appris, le matériel de ses productions linguistiques futures. L'analogie offre au locuteur les moyens fiables de produire dans la langue, car les seuls disponibles à ses yeux et qui ont, pour certains, antérieurement prouvé leur efficacité. L'analogie est ainsi au cœur de productions linguistiques, elle permet de résoudre les problèmes qui se présentent au locuteur.

Le caractère novateur, notamment à travers la néologie et l'introduction de nouvelles formes linguistiques, renforce le recours à l'analogie et lui accorde une position centrale dans la cognition humaine, et par là, l'appropriation-production linguistique. Le locuteur construit, dans une première phase, une connaissance générale en exploitant la pertinence du contenu de la source, puis dans une seconde phase, une connaissance particulière de la cible qui intègre les propriétés de la source. L'accroissement des connaissances fera que le locuteur passera d'une généralisation à une différenciation des savoirs. Celle-ci sera jugée plus appropriée en fonction du contexte d'apparition ou d'utilisation du savoir linguistique. Toute nouvelle connaissance s'intègre aux acquis antérieurs et vient les renforcer, les enrichir et les modifier. Chaque nouvelle connaissance dépend de celles acquises, elle modifie celles qui existent, et sera elle-même modifiée ultérieurement par les nouveaux acquis. Plus une forme linguistique est utilisée, plus elle est reproduite; plus une forme est récurrente dans les discours, plus elle sert de base 
à la construction de nouvelles formes et à la modification de celles antérieurement acquises. Les connaissances s'adaptent, changent et se modifient au contact des autres savoirs dans différents contextes de communication. Le processus analogique aide à les organiser et à les produire.

L'homme est ainsi un être analogique. S'intéresser à l'analogie dynamique, celle par laquelle la langue évolue et se diversifie, c'est mieux comprendre le fonctionnement cognitif du locuteur lors de son appropriation-production linguistique. La création linguistique est le résultat du calcul d'une quatrième proportionnelle qui est inconnue du locuteur. Celui-ci déduit l'inconnue du rapport entre les deux premiers éléments de l'analogie de manière à avoir un rapport similaire entre les troisième et quatrième éléments. Ce serait de cette manière que le locuteur agirait lorsqu'il crée une nouvelle unité linguistique qu'il ignore. Cela fait que l'analogie est au cœur du comportement langagier du locuteur et de l'organisation du système linguistique.

Cette contribution pourrait connaître des prolongements qui démontreraient notamment les failles des calculs analogiques malgré un apprentissage structurel et systémique de la langue, et détermineraient si le processus analogique se réalise de la même manière chez le locuteur souffrant de troubles et de pathologies du langage (les enfants autistes, par exemple). Ces études seraient d'un apport considérable à la linguistique, et apporteraient des solutions, ou du moins des réponses, à certaines questions d'appropriation d'un autre ordre.

\section{Références bibliographiques}

ARISTOTE (1980, [rédigé vers 335 avant J.-C.]). La Poétique, Paris. Seuil.

BAJRIĆ, S. (2013 [2009]). Linguistique, cognition et didactique : principes et exercices de linguistique-didactique. Paris. PUPS.

BANGE, P. (2005). L'apprentissage d'une langue étrangère: cognition et interaction. Paris. L'Harmattan.

BASSANO, D. (1998b), Sémantique et syntaxe dans l'acquisition des classes de mots : l'exemple des noms et des verbes en français, Langue Française, n 118 , p. 26-48.

BIJELJAC-BABIC, R. (2000), Acquisition de la phonologie et bilinguisme précoce, dans KAIL, M., L'acquisition du langage, Paris, PUF, p. 169-192.

BLANCHE-BENVENISTE, C. (1990). Le français parlé. Paris. Editions du CNRS.

BLOOMFIELD, L. (1970 [1933]). Langage, traduction de Gazio J., Paris. Payot.

BOUGCHICHE, R. (2008). Le français parlé des immigrés kabylophones en région parisienne, Paris-Sorbonne (Paris IV). Mémoire de master 2, (non publié).

BOUGCHICHE, R. (2013). L'analogie dans l'apprentissage des langues. Thèse de doctorat, Paris-Sorbonne (Paris IV).

BOUTON, Ch. P. (1969). Mécanismes d'acquisition du français langue étrangère chez l'adulte. Paris. Klincksieck.

CLARK, E. V. (1998), Lexique et syntaxe dans l'acquisition du français, Langue Française, $\mathrm{n}^{\circ}$ 118, p. 49-60.

DAL, G. (2003). Productivité morphologique : définitions et notions connexes. Langue française, n 140, p. 03-23.

DE HEREDIA, Ch. (1973), Étude du langage d'une enfant de quatre ans, en milieu immigrant espagnol à Paris, processus d'acquisition: le rôle de l'analogie et des situations de communication, Thèse de doctorat, ParisSorbonne (Paris V), Université René Descartes. 
DERWIN, B. L. \& SKOUSEN, R. (1989). Real-time morphology: symbolic rules or analogical networks? Proceedings of the fifteenth annual meeting of the Berkeley Linguistics Society, p. 48-62.

EL KILANI, J. (1983), Le français parlé par les immigrés marocains en France, Thèse de doctorat, Paris-Sorbonne (Paris V), Université René Descartes.

FREI, H., (1993 [1929]). La grammaire des fautes, Genève-Paris. Slatkine Reprints.

GENTNER, D. (1983). Structure-mapping: A theoretical framework for analogy», Cognitive Science, $\mathrm{n}^{\circ}$ 7, p.155170.

GENTNER, D. (1989). The mechanisms of analogical learning, dans VOSNIADOU, S. \& ORTONY, A., Similarity and analogical reasoning, New York, Cambridge University Press, p. 197-241.

GENTNER, D. \& COLHOUN, J. (2010). Analogical processes in human thinking and learning, dans GLATZDER, B. M., GOEL, V. \& MÜLLER, A. von (eds.)., On thinking, vol. 2, Towards a theory of thinking, Berlin Heidelberg, Springer-Verlag, p. 35-48.

GOSWAMI, U. (1992). Analogical reasoning in children. East Sussex. Lawrence Erlbaum Associates.

GREGOIRE, A. (1947). L'apprentissage du langage II. La troisième année et les années suivantes, Bruxelles. Duculot.

GUILLAUME, G. (1982). Leçons de linguistique 1956-1957, Québec, PUL.

HILAIRE-DEBOVE, G. \& KERN, S. (2003), Le rôle de l'analogie dans l'acquisition de la langue maternelle, dans Regards croisés sur l'analogie, Paris, Lavoisier, p. 775-786.

HOLYOAK, K. J. \& THAGARD, P. (1995). Mental Leaps, Analogy in creative thought. Cambridge. A Bradford Book. The MIT Press.

HUMBOLDT, W. V. (2000). Sur le caractère national des langues, Paris. Seuil.

ITKONEN, E. \& HAUKIOJA, J. (1997). A rehabilitation of analogy in syntax (and elsewhere). Metalinguistik im Wandel: die kognitive Wende in Wissenschaftstheorie und Linguistik, Francfurt, A. Kertesz edition, p. 131-177.

ITKONEN, E. (2005). Analogy as structure and process. Amsterdam. John Benjamins Publishing Company.

LAVIE, R.-J. (2003). Le locuteur analogique ou la grammaire mise à sa place, Université Paris X Nanterre. Thèse de doctorat.

LAVIE, R.-J. (2004). Systemic productivity must complement structural productivity. Linguagem, Cultura e Cognição: Estudos de Linguística Cognitiva, Coimbra, Editions Almedina.

MARCHELLO-NIZIA, Ch. (2006). Grammaticalisation et changement linguistique. Bruxelles. Champs linguistiques, De Boeck \& Larcier.

MONNERET, Ph. (2004). Essais de linguistique analogique. Dijon. ABELL.

MORSLY, D. (1983). Interférence de l'arabe sur le français de travailleurs immigrés à Paris, thèse de doctorat, Paris-Sorbonne (Paris V), Université René Descartes.

PAUL, H., (1920 [1880]), Prinzipien der Sprachgeschichte. Tübingen, Niemeyer.

PONCHON, Th. (2007). Analogie, diachronie et psychomécanique du langage. Dijon. Cahiers de linguistique analogique, ABELL, p. 29-67.

QUEFFÉLEC A. et al. (2002). Le français en Algérie : lexique et dynamique des langues, Bruxelles. Éditions Duculot.

SAUSSURE, F. de (1946 [1916]). Cours de linguistique générale. Paris. Payot.

SIOUFFI, G. (2010). Penser le langage à l'âge classique. Paris. Armand Colin.

TOMASELLO, M. (2003). Constructing a Language. A Usage-Based Theory of Language Acquisition. Boston. Harvard University Press.

Dictionnaire de l'Académie française, (1798). $5^{\mathrm{e}}$ édition. 


\begin{abstract}
${ }^{1}$ Nous sommes en train de constituer un corpus de productions authentiques et spontanées du français de touristes germanophones, hispanophones et lusophones apprenant le français. Cette collecte est réalisée en milieu touristique (hôtellerie). Elle a servi à exemplifier certains éléments de la présente étude.
\end{abstract}

${ }^{2}$ A cause de leur appréhension et pour que les productions soient spontanées, nous avons enregistré les «primomigrants » à leur insu. A chaque fin d'enregistrement, nous les avons mis au courant de notre projet (préparation du mémoire de master2) et du fait de les avoir enregistrés. Ils ne l'ont pas mal pris. Les «jeunes-migrants » étaient au courant de l'enregistrement dès le départ, car ils n'étaient pas réticents à ce genre d'expérience.

${ }^{3}$ Il existe plusieurs types d'analogies linguistiques, notamment binaire, mais celle qui nous intéresse dans cette étude est celle développée depuis l'Antiquité, notamment par Pythagore et Aristote qui s'intéressent à la relation entre les composants de l'analogie.

${ }^{4}$ Cité par SIOUFFI, G., 2010, p. 35.

${ }^{5}$ Ibidem., p. 36.

${ }^{6}$ Exemple emprunté à LAVIE, R.-J., 2003, p. 342. Cela dit, cette analogie est parfaitement sémantique si les deux protagonistes sont frères et/ou sœurs, ce qui n'est pas exprimé dans cet exemple.

${ }^{7}$ LAVIE, R.-J., 2003, p. 170. Un rapprochement peut être fait entre la position de Guillaume et celle de Humboldt (2000, p. 99). Ce dernier voit dans la formation de nouveaux sons l'action de l'analogie.

${ }^{8}$ Il existe un adjectif dérivé de champignon : champignonneux, mais fongique sert aussi d'adjectif synonymique dans un cadre médical, par exemple. Fongique est dérivé de fungus.

${ }^{9}$ BLOOMFIELD, L., 1970, p. 258 (traduction de GAZIO, J.).

10 Tomasello (2003 : 295) distingue quatre processus intervenants dans la construction du langage et que chacun assume une fonction particulière: intention-reading and cultural learning; schematization and analogy ; entrenchment and competition; functionally based distributional analysis.

${ }^{11}$ LAVIE, R.-J., 2003, p. 103. Nous n'entrons pas dans le détail de cette opposition que fait Lavie. La thèse de Lavie (2003) comporte un panel de démonstrations sur les différents types d'analogies et des testes de fonctionnements, et de certains calculs analogiques très intéressants.

${ }^{12}$ La combinaison des constructions structurelles et systémiques n'épargnent pas la réalisation d'erreurs linguistiques. Le locuteur est toujours susceptible de construire des analogies hasardeuses, non conformes aux pratiques linguistiques de la communauté qui parle la langue en question, même lorsqu'il s'agit d'un locuteur natif et naïf dont l'intuition linguistique est très développée (exemple de *bravitude produit par une candidate à l'élection présidentielle de la France de 2007).

${ }^{13}$ Cité par BANGE, P., 2005, p. 60.

${ }^{14}$ Le morphème -eur est très productif en français (de même qu'en anglais -or). On observe cette caractéristique dans l'usage qu'en font les enfants dans leur construction lexicale en $X$ (nom ou racine verbale) + -eur (Clark 1998).

15 Terme utilisé pour désigner un jeune chômeur adossé à un mur à ne rien faire.

16 Terme utilisé pour désigner une personne qui s'occupe des affaires du quartier qu'elle ne quitte jamais.

${ }^{17}$ Les mêmes remarques valent pour d'autres locuteurs qui apprennent le français et dont la langue maternelle est autre que le kabyle, le russe par exemple.

${ }^{18}$ En kabyle, métro est intégré de deux manières : amitro qui correspond à la forme des noms masculins singuliers dont l'initiale est $a$-, et lmitro qui est une variante récente de le métro, notamment chez les kabylophones de France. Cette dernière forme reprend l'intégration en kabyle des emprunts au français avec l'article défini agglutiné au nom qu'il définit (comme dans lamiri « la mairie », lmarchi « le marché », etc.).

${ }^{19}$ Cet exemple doit être contextualisé. Il s'agit d'une situation où un père demande à son enfant de lui prendre la main (ou tenir la main) en marchant dans la rue. L'usage du verbe attraper est dû à la version kabyle : tef afusiw [țəf/afusiw] «attrape ma main ».

${ }^{20}$ Si l'on considère la création du point de vue de la norme, de l'évolution du système linguistique qui intègre et/ou refuse les créations des locuteurs, elle s'inscrit dans un cadre diachronique. 
${ }^{21}$ Ces deux derniers exemples sont fréquents chez les francophones natifs.

${ }^{22}$ Ces deux verbes partagent la même marque à l'infinitif, mais ils intègrent deux groupes différents. Le marquage infinitif est également une source d'erreurs, surtout pour la formation du futur pour le verbe aller qui donne des formes comme *allera.

${ }^{23}$ Exemple produit par une touriste lusophone comme suit : «vous avez un sécheur » avec le geste de la main vers la tête.

${ }^{24}$ Cet exemple a été produit comme suit : «aujourd'hui, *le pluie très fort » pour «aujourd'hui, la pluie est très forte ». 